\title{
ON INDUCTION OF CLASS FUNCTIONS
}

\author{
G. LUSZTIG
}

\begin{abstract}
Let $G$ be a connected reductive group defined over a finite field $\mathbf{F}_{q}$ and let $L$ be a Levi subgroup (defined over $\mathbf{F}_{q}$ ) of a parabolic subgroup $P$ of $G$. We define a linear map from class functions on $L\left(\mathbf{F}_{q}\right)$ to class functions on $G\left(\mathbf{F}_{q}\right)$. This map is independent of the choice of $P$. We show that for large $q$ this map coincides with the known cohomological induction (whose definition involves a choice of $P$ ).
\end{abstract}

\section{INTRODUCTION}

0.1 . Let $\mathrm{G}$ be a connected reductive algebraic group over $\mathbf{k}$, an algebraic closure of the finite field $F_{q}$ with $q$ elements, with a fixed $F_{q}$-rational structure whose Frobenius map is denoted by $F: G \rightarrow G$.

Let $\Lambda(G)$ be the set of subgroups $M$ of $G$ such that $M$ is a Levi subgroup of a parabolic subgroup of $G$; for $M \in \Lambda(G)$ let $\Pi(M)$ be the set of parabolic subgroups $P$ of $G$ for which $M$ is a Levi subgroup. Assume that $M \in \Lambda(G)$ is defined over $F_{q}$ and that $P \in \Pi(M)$ (not necessarily defined over $\left.F_{q}\right)$. Let $\mathcal{K}\left(G^{F}\right)\left(\operatorname{resp} . \mathcal{K}\left(M^{F}\right)\right.$ ) be the Grothendieck group of representations of $G^{F}$ (resp. $M^{F}$ ) over an algebraic closure $\overline{\mathbf{Q}}_{l}$ of the $l$-adic numbers where $l$ is a prime number not dividing $q$. (When $F$ acts on a set $X$ we denote by $X^{F}$ the fixed point set of $F: X \rightarrow X$.) Let $R_{M, P}^{G}: \mathcal{K}\left(M^{F}\right) \rightarrow \mathcal{K}\left(G^{F}\right)$ be the "induction" homomorphism defined in DL (in the case where $M$ is a maximal torus) and in [L76] (in the general case). Let $\operatorname{cl}\left(G^{F}\right)$ (resp. $c l\left(M^{F}\right)$ ) be the $\overline{\mathbf{Q}}_{l}$-vector space of class functions $G^{F} \rightarrow \overline{\mathbf{Q}}_{l}$ (resp. $\left.M^{F} \rightarrow \overline{\mathbf{Q}}_{l}\right)$. By passage to characters, $R_{M, P}^{G}$ can be regarded as a $\overline{\mathbf{Q}}_{l}$-linear map $R_{M, P}^{G}: \operatorname{cl}\left(M^{F}\right) \rightarrow \operatorname{cl}\left(G^{F}\right)$. In [L76] it was conjectured that

(a) $R_{M, P}^{G}$ is independent of the choice of $P$.

(At that time it was already known from $\mathrm{DL}$ that (a) holds when $M$ is a maximal torus of $G$, so that in that case, the notation $R_{M}^{G}$ can be used instead of $R_{M, P}^{G}$.) As noted in [L76], Deligne had an argument to prove (a) for any $M$ provided that $q \gg 0$ (but his proof has not been published). In [L90, 8.13] a proof of (a) for $q \gg 0$ was given which was based on the theory of character sheaves and thus was quite different from Deligne's proof. (In loc. cit. there is also an assumption on the characteristic $p$ of $\mathbf{k}$, but that assumption can be removed in view of the cleanness result for character sheaves in [L12.) In [BM] it is proved that (a) holds assuming only that $q>2$.

Received by the editors July 31, 2020, and, in revised form, November 27, 2020, and December 4, 2020.

2020 Mathematics Subject Classification. Primary 20 G99.

This research was supported by NSF grant DMS-1855773. 
In this paper we define a $\overline{\mathbf{Q}}_{l}$-linear map $\mathcal{R}_{M}^{G}: \operatorname{cl}\left(M^{F}\right) \rightarrow \operatorname{cl}\left(G^{F}\right)$ with no restriction on $q$ (see 1.7) and we show that

(b) if $q \gg 0$ we have $\mathcal{R}_{M}^{G}=R_{M, P}^{G}$ for any $P \in \Pi(M)$.

(See 1.9] and 92). We expect that the results of [L90] quoted in this paper hold without restriction on $q$ and, as a consequence, that (b) holds without restriction on $q$.

The definition of $\mathcal{R}_{M}^{G}$ is in terms of intersection cohomology; it relies on ideas of L84. The proof of (b) relies on the results of [L90 connecting representations of $G^{F}$ with the character sheaves on $G$. In $₫ 3$ we show (based on results of [L90]) that if $D$ is an $F$-stable conjugacy class of $G^{F}$ then the function on $G^{F}$ which is 1 on $D^{F}$ and 0 on $G^{F}-D^{F}$ is a linear combination of characters of $R_{T}^{G}(\theta)$ for various $F$-stable maximal tori of $G$ and various characters $\theta$ of $T^{F}$. (This was conjectured in [L78.)

0.2. Notation. Let $\nu_{G}$ be the dimension of the flag manifold of $G$. Let $\mathcal{Z}_{G}$ be the centre of $G$. For $M \in \Lambda(G)$ let $N_{G} M$ be the normalizer of $M$ in $G$. For $g \in G$ we have $g=g_{s} g_{u}=g_{u} g_{s}$ where $g_{s} \in G$ is semisimple and $g_{u} \in G$ is unipotent. For $s \in G$ semisimple we write $G_{s}$ for the centralizer of $s$ in $G$. For $g \in G$ let $H_{G}(g)$ be the smallest subgroup in $\Lambda(G)$ that contains $G_{g_{s}}^{0}$. If $G^{\prime}$ is a subgroup of $G$, let $Z_{G}\left(G^{\prime}\right)$ be the centralizer of $G^{\prime}$ in $G$. Let $G_{d e r}$ be the derived subgroup of $G$.

Let $X$ be an algebraic variety over $\mathbf{k}$. Let $l s(X)$ be the collection of $\overline{\mathbf{Q}}_{l}$-local systems on $X$. If $H$ is a connected algebraic group acting on $X$ we denote by $l s_{H}(X)$ the collection of $H$-equivariant $\overline{\mathbf{Q}}_{l}$-local systems on $X$. Let $Y$ be a locally closed, smooth, irreducible subvariety of $X$ and let $\mathcal{E} \in l s(Y)$. Then $\mathcal{E}$ extends canonically as an intersection cohomology complex to the closure $\bar{Y}$ of $Y$ and to $X$ by 0 on $X-\bar{Y}$; the resulting complex on $X$ is denoted by $\mathcal{E}^{\sharp}$. Assume now that $X$ is defined over $F_{q}$ with Frobenius map $F: X \rightarrow X$ and $Y$ above is $F$-stable. Assume that $F^{*} \mathcal{E} \cong \mathcal{E}$ and we are given an isomorphism $\phi: F^{*} \mathcal{E} \stackrel{\sim}{\longrightarrow} \mathcal{E}$. This induces an isomorphism $\phi^{\sharp}: F^{*} \mathcal{E}^{\sharp} \stackrel{\sim}{\rightarrow} \mathcal{E}^{\sharp}$. Let $\chi_{\mathcal{E}, \phi}: X^{F} \rightarrow \overline{\mathbf{Q}}_{l}$ be the function whose value at $y \in Y^{F}$ is the trace of $\phi$ on the stalk of $\mathcal{E}$ at $y$ and which is zero on $X^{F}-Y^{F}$ Let $\chi_{\mathcal{E}^{\sharp}, \phi^{\sharp}}: X^{F} \rightarrow \overline{\mathbf{Q}}_{l}$ be the function whose value at $x \in X^{F}$ is the alternating sum of traces of the linear maps induced by $\phi^{\sharp}$ on the stalks at $x$ of the cohomology sheaves of $\mathcal{E}^{\sharp}$. We have $\chi_{\mathcal{E}, \phi}\left|Y^{F}=\chi_{\mathcal{E}^{\sharp}, \phi^{\sharp}}\right| Y^{F}$.

\section{The Definition of $\mathcal{R}_{M}^{G}$}

1.1. The conjugacy class of $g \in G$ is said to be isolated if $H(g)=G$. A subset $S$ of $G$ is said to be an isolated stratum if $S$ is the inverse image of an isolated conjugacy class of $G / \mathcal{Z}_{G}^{0}$ under the obvious map $G \rightarrow G / \mathcal{Z}_{G}^{0}$. Let $A_{G}$ be the set of pairs $(L, S)$ where $L \in \Lambda(G)$ and $S$ is an isolated stratum of $L$. For $(L, S) \in A_{G}$ we set $S_{r}^{G}=\left\{g \in S ; H_{G}(g)=L\right\}=\left\{g \in S ; G_{g_{s}}^{0} \subset L\right\}$ and $Y_{L, S}^{G}=\cup_{x \in G} x S_{r}^{G} x^{-1}$. Then

(a) $Y_{L, S}^{G}$ is a smooth locally closed irreducible subvariety of $G$ of dimension $2 \nu_{G}-$ $2 \nu_{L}+\operatorname{dim} S$.

(see [L84, 3.1]). The subsets $Y_{L, S}^{G}$ are called the strata of $G$. We have

$$
G=\sqcup_{(L, S) \in A_{G}} \text { up to G-conjugacy } Y_{L, S}^{G} .
$$

Note that an isolated stratum $S$ of $G$ is the stratum $Y_{G, S}^{G}$. 
1.2. Let $M \in \Lambda(G)$. Let $Y^{\prime}$ be a stratum of $M$. We shall associate to $Y^{\prime}$ a stratum $Y$ of $G$ as follows.

We set $Y=Y_{L, S}^{G}$ where $(L, S) \in A_{M}$ is such that $Y^{\prime}=Y_{L, S}^{M}=\cup_{x \in M} x S_{r}^{M} x^{-1}$. (We have also $(L, S) \in A_{G}$.) We set $Y_{r}^{\prime}=\cup_{x \in M} x S_{r}^{G} x^{-1}$.

Now $Y_{r}^{\prime}$ and $Y$ are independent of the choice of $(L, S)$. (Indeed, it is enough to show that if $m \in M$, then $\left(m S m^{-1}\right)_{r}^{G}=m S_{r}^{G} m^{-1}$; we use that $H_{G}\left(m g m^{-1}\right)=$ $m H_{G}(g) m^{-1}$.) We have $Y_{r}^{\prime} \neq \emptyset, Y_{r}^{\prime} \subset Y$ and $Y$ is the unique stratum of $G$ that contains $Y_{r}^{\prime}$. We have also $Y_{r}^{\prime} \subset Y^{\prime}$. (We use that $S_{r}^{G} \subset S_{r}^{M}$; indeed, if $g \in S$ and $H_{G}(g)=L$, then $G_{g_{s}}^{0} \subset L$ hence $M_{g_{s}}^{0} \subset L$ and $H_{M}(g)=L$.) We show that

(a) $Y_{r}^{\prime}$ is open in $Y^{\prime}$.

Let $(L, S) \in A_{M}$ be such that $Y^{\prime}=Y_{L, S}^{M}$. Now $Y^{\prime}$ is a locally trivial fibration over the variety of all $M$-conjugates of $L$, via $g \mapsto H_{M}(g)$; let $\beta$ be the fibre of this map over $L$. It is enough to show that $Y_{r}^{\prime} \cap \beta$ is open in $\beta$. We have $\beta=\cup_{n \in N_{M} L / L} n S_{r}^{M} n^{-1}, Y_{r}^{\prime} \cap \beta=\cup_{n \in N_{M} L / L} n S_{r}^{G} n^{-1}$. It is enough to observe that $S_{r}^{G}$ is open in $S_{r}^{M}$; in fact it is open in $S$.

Let $\tilde{\tilde{Y}}=\left\{(g, x) \in G \times G ; x^{-1} g x \in Y_{r}^{\prime}\right\}$. Define $\sigma: \tilde{\tilde{Y}} \rightarrow Y_{r}^{\prime}$ by $\sigma(g, x)=x^{-1} g x$. Let $\tilde{Y}=\tilde{\tilde{Y}} / M=\left\{(g, x M) \in Y \times G / M ; x^{-1} g x \in Y_{r}^{\prime}\right\}$. We show:

(b) $\tilde{Y}$ is a smooth, irreducible variety of dimension equal to $\operatorname{dim} Y$.

Since $Y_{r}^{\prime}$ is smooth, irreducible of dimension equal to $\operatorname{dim} Y^{\prime}$ (see (a), 1.1(a)), we see that $\tilde{Y}$ is smooth, irreducible of dimension $\operatorname{dim} G / M+\operatorname{dim} Y^{\prime}=\operatorname{dim} Y$. This proves (b).

If $(g, x M) \in \tilde{Y}$, we have $g \in Y$ (since $\left.Y_{r}^{\prime} \subset Y\right)$. Define $\pi: \tilde{Y} \rightarrow Y$ by $(g, x M) \mapsto$ $g$. We show:

(c) $\pi$ is a finite unramified cover of $Y$ with fibres isomorphic to $c_{G} / c_{M}$ where $c_{G}=\left\{n \in N_{G} L, n^{-1} S n=S\right\}, c_{M}=\left\{n \in N_{M} L, n^{-1} S n=S\right\}$ and $(L, S) \in A_{M}$, $Y^{\prime}=Y_{L, S}^{M}$.

It is enough to show that if $g \in S_{r}^{G}$, then $\pi^{-1}(g) \cong c_{G}$. We can identify $\pi^{-1}(g)$ with $\left\{x M \in G / M ; x^{-1} g x \in Y_{r}^{\prime}\right\}$ hence also with $\left\{x \in G ; \xi^{-1} g x \in S_{r}^{G}\right\} /\{x \in$ $\left.M ; \xi^{-1} g x \in S_{r}^{G}\right\}$. It is enough to show that $\left\{x \in G ; \xi^{-1} g x \in S_{r}^{G}\right\}=c_{G}$ (this would imply $\left.\left\{x \in M ; \xi^{-1} g x \in S_{r}^{G}\right\}=c_{M}\right)$. Let $x \in G$ be such that $x^{-1} g x \in S_{r}^{G}$; then $L=H_{G}\left(x^{-1} g x\right)=x^{-1} H_{G}(g) x=x^{-1} L x$ and $x \in N_{G} L$. Let $S^{\prime}, g^{\prime}$ be the image of $S, g$ in $L / \mathcal{Z}_{L}^{0}$. Now $A d\left(x^{-1}\right)$ induces an automorphism of $L / \mathcal{Z}_{L}^{0}$ which carries $S^{\prime}$ to an isolated conjugacy class $S^{\prime \prime}$ and $g^{\prime} \in S^{\prime}$ to an element of $S^{\prime}$; it follows that $S^{\prime}=S^{\prime \prime}$ and $\operatorname{Ad}\left(x^{-1}\right) S=S$, so that $x \in c_{G}$. Conversely, let $x \in N_{G} L$ be such that $x^{-1} S x=S$. Then $x^{-1} g x \in S, H_{G}\left(x^{-1} g x\right)=x^{-1} H_{G}(g) x=x^{-1} L x=L$ and $x^{-1} g x \in S_{r}^{G}$. This proves (c).

1.3. We preserve the setup of 1.2 Let $\mathcal{E} \in l s_{M}\left(Y^{\prime}\right)$. We define $j_{Y^{\prime}}^{Y}(\mathcal{E}) \in l s_{G}(Y)$. Note that $\sigma^{*}\left(\mathcal{E} \mid Y_{r}^{\prime}\right) \in l s_{G \times M}(\tilde{\tilde{Y}})$ for the $G \times M$ action $\left(g_{0}, m\right):(g, x) \mapsto\left(g_{0} g g_{0}^{-1}\right.$, $\left.g_{0} x m^{-1}\right)$ on $\tilde{\tilde{Y}}$. Hence $\sigma^{*}\left(\mathcal{E} \mid Y_{r}^{\prime}\right)=\sigma_{1}^{*} \mathcal{E}_{1}$ where $\sigma_{1}: \tilde{\tilde{Y}} \rightarrow \tilde{Y}$ is the obvious map and $\mathcal{E}_{1} \in l s(\tilde{Y})$ is well defined. We define $j_{Y^{\prime}}^{Y} \mathcal{E}=\pi_{*}\left(\mathcal{E}_{1}\right) \in l s_{G}(Y)$; this has rank equal to $c_{G} / c_{M}$ times the rank of $\mathcal{E}$.

1.4. We preserve the setup of 1.3. Let $(L, S) \in A_{M}$ be such that $Y^{\prime}=Y_{L, S}^{M}$ and let $\mathcal{E}_{0} \in l s_{L}(S)$. Then $j_{S}^{Y^{\prime}}\left(\mathcal{E}_{0}\right) \in l s\left(Y^{\prime}\right)$ and $j_{S}^{Y}\left(\mathcal{E}_{0}\right) \in l s(Y)$ are defined as in 1.3 . From the definition we see that

(a) $j_{Y^{\prime}}^{Y}\left(j_{S}^{Y^{\prime}} \mathcal{E}_{0}\right)=j_{S}^{Y}\left(\mathcal{E}_{0}\right)$. 
1.5. Let $\mathcal{F} \in l s_{G}\left(Y_{1}\right)$ where $Y_{1}=Y_{L, S}^{G}$. We say that $\mathcal{F}$ is admissible if it is irreducible and if $\mathcal{F}$ is a direct summand of $j_{S}^{Y_{1}}\left(\mathcal{F}_{0}\right) \in l s_{G}\left(Y_{1}\right)$ for some $\mathcal{F}_{0} \in l s_{L}(S)$ which is cuspidal irreducible (see [L84, 2.4]). (This condition is independent of the choice of $(L, S)$.) Let $\mathcal{A}^{G}\left(Y_{1}\right)$ be the class of $G$-equivariant admissible local systems on $Y_{1}$. We say that $Y_{1}$ is an admissible stratum if $\mathcal{A}^{G}\left(Y_{1}\right) \neq \emptyset$. In the setup of 1.3 we see (using 1.4 (a)) that if $\mathcal{F} \in \mathcal{A}^{M}\left(Y^{\prime}\right)$ then $j_{Y^{\prime}}^{Y}(\mathcal{F})$ is a (nonzero) direct sum of objects of $\mathcal{A}^{G}(Y)$. In particular, if $Y^{\prime}$ is admissible (for $M$ ) then $Y$ is admissible (for $G$ ).

1.6. We now assume that $M$ is defined over $F_{q}$. If $Y^{\prime}$ in 1.2 is $F$-stable then $Y_{r}^{\prime}$ in 1.2 is $F$-stable. Indeed, from $Y^{\prime}=F\left(Y^{\prime}\right)$ and $Y^{\prime}=Y_{L, S}^{M}$ we deduce $Y^{\prime}=F\left(Y^{\prime}\right)=$ $Y_{F(L), F(S)}^{M}$ hence $F(L)=m L m^{-1}, F(S)=m S m^{-1}$ for some $m \in M$. Replacing $L, S$ by an $M$-conjugate we can assume that $F(L)=L, F(S)=S$, so that

$$
F\left(Y_{r}^{\prime}\right)=\cup_{x \in M} x F(S)_{r}^{G} x^{-1}=\cup_{x \in M} x S_{r}^{G} x^{-1}=Y_{r}^{\prime} .
$$

A similar argument shows that $Y$ in 1.2 is $F$-stable; alternatively, this holds since $Y$ is the unique stratum of $G$ containing $Y_{r}^{\prime}$ (which is $F$-stable). Moreover, $\tilde{\tilde{Y}}, \tilde{Y}, \pi$ in 1.2 and $\sigma, \sigma_{1}$ in 1.3 are defined over $F_{q}$. If now $\mathcal{E}$ in 1.3 is such that $F^{*} \mathcal{E} \cong \mathcal{E}$, then $\sigma^{*}\left(\mathcal{E} \mid Y_{r}^{\prime}\right)$ and $\mathcal{E}_{1}$ in 1.3 are isomorphic to their inverse image under $F$ hence $\tilde{\mathcal{E}}:=j_{Y^{\prime}}^{Y}(\mathcal{E})$ satisfies $F^{*} \tilde{\mathcal{E}} \cong \tilde{\mathcal{E}}$; moreover any isomorphism $\phi: F^{*} \mathcal{E} \stackrel{\sim}{\longrightarrow} \mathcal{E}$ (of local systems on $\left.Y^{\prime}\right)$ induces an isomorphism $F^{*}\left(\sigma^{*}\left(\mathcal{E} \mid Y_{r}^{\prime}\right)\right) \stackrel{\sim}{\longrightarrow} \sigma^{*}\left(\mathcal{E} \mid Y_{r}^{\prime}\right)$ (of local systems on $\tilde{\tilde{Y}}$ ), an isomorphism $F^{*} \mathcal{E}_{1} \stackrel{\sim}{\longrightarrow} \mathcal{E}_{1}$ (of local systems on $\tilde{Y}$ ) and an isomorphism $\tilde{\phi}: F^{*} \tilde{\mathcal{E}} \cong \tilde{\mathcal{E}}$ (of local systems on $Y$ ). Now $\phi, \tilde{\phi}$ extend to isomorphisms $\phi^{\sharp}$ : $F^{*} \mathcal{E}^{\sharp} \stackrel{\sim}{\longrightarrow} \mathcal{E}^{\sharp}$ (of complexes on $M$ ) and $\tilde{\phi}^{\sharp}: F^{*} \tilde{\mathcal{E}}^{\sharp} \stackrel{\sim}{\longrightarrow} \tilde{\mathcal{E}}^{\sharp}$ (of complexes on $G$ ). Hence $\chi_{\mathcal{E}^{\sharp}, \phi^{\sharp}}: M^{F} \rightarrow \overline{\mathbf{Q}}_{l}, \chi_{\tilde{\mathcal{E}}^{\sharp}, \tilde{\phi}^{\sharp}}: G^{F} \rightarrow \overline{\mathbf{Q}}_{l}$ are well defined. They are class functions on $M^{F}$ (resp. $\left.G^{F}\right)$.

1.7. Let $Z_{M}$ be the set of all pairs $\left(Y^{\prime}, \mathcal{E}\right)$ where $Y^{\prime}$ is an $F$-stable admissible stratum of $M$ and $\mathcal{E} \in l s_{M}\left(Y^{\prime}\right)$ is admissible (up to isomorphism) such that $F^{*} \mathcal{E} \cong \mathcal{E}$. For any $\left(Y^{\prime}, \mathcal{E}\right) \in Z_{M}$ we denote by $\mathcal{L}_{Y^{\prime}, \mathcal{E}}$ the subspace of $\operatorname{cl}\left(M^{F}\right)$ consisting of the class functions $\chi_{\mathcal{E}^{\sharp}, \phi^{\sharp}}: M^{F} \rightarrow \overline{\mathbf{Q}}_{l}$ where $\phi: F^{*} \mathcal{E} \stackrel{\sim}{\rightarrow} \mathcal{E}$ is an isomorphism. A different choice for $\phi$ must be of the form $a \phi$ for some $a \in \overline{\mathbf{Q}}_{l}^{*}$ and we have $\chi_{\mathcal{E}^{\sharp},(a \phi)^{\sharp}}=a \chi_{\mathcal{E}^{\sharp}, \phi^{\sharp}}$ hence $\mathcal{L}_{Y^{\prime}, \mathcal{E}}$ is well defined line in $\operatorname{cl}\left(M^{F}\right)$, independent of any choice. We have

(a) $\operatorname{cl}\left(M^{F}\right)=\oplus_{\left(Y^{\prime}, \mathcal{E}\right) \in Z_{M}} \mathcal{L}_{Y^{\prime}, \mathcal{E}}$.

A proof is given in [L04, 26.5]. (Alternatively, instead of [L04, one can use [L86, 25.2] complemented by [L12].) Let

(b) $\mathcal{R}_{M}^{G}: \operatorname{cl}\left(M^{F}\right) \rightarrow \operatorname{cl}\left(G^{F}\right)$

be the linear map such that for any $\left(Y^{\prime}, \mathcal{E}\right) \in Z_{M}$, the restriction of $\mathcal{R}_{M}^{G}$ to the line $\mathcal{L}_{Y^{\prime}, \mathcal{E}}$ sends $\chi_{\mathcal{E}^{\sharp}, \phi^{\sharp}}: M^{F} \rightarrow \overline{\mathbf{Q}}_{l}$ (where $\phi: F^{*} \mathcal{E} \stackrel{\sim}{\rightarrow} \mathcal{E}$ is an isomorphism) to $\chi_{\tilde{\mathcal{E}}_{\sharp}^{\sharp}, \tilde{\phi}^{\sharp}}: G^{F} \rightarrow \overline{\mathbf{Q}}_{l}$ (see 1.6). If $\phi$ is replaced by $a \phi$ with $a \in \overline{\mathbf{Q}}_{l}^{*}$, then $\tilde{\phi}^{\sharp}$ is replaced by $a \tilde{\phi}^{\sharp}$. Thus the linear map $\mathcal{R}_{M}^{G}$ is well defined (independent of choices).

1.8. In the setup of 1.4 assume that $\mathcal{E}_{0} \in l s_{L}(S)$ is cuspidal irreducible, that $L, S$ are defined over $F_{q}$ and that we are given an isomorphism $\phi_{0}: F^{*} \mathcal{E}_{0} \stackrel{\sim}{\longrightarrow} \mathcal{E}_{0}$ of local systems on $S$. Then $\chi_{\mathcal{E}_{0}^{\sharp}, \phi_{0}^{\sharp}}: L^{F} \rightarrow \overline{\mathbf{Q}}_{l}$ is well defined. Using 1.4(a) and the definitions we see that

(a) $\left.\mathcal{R}_{M}^{G}\left(\mathcal{R}_{L}^{M}\left(\chi_{\mathcal{E}_{0}^{\sharp}, \phi_{0}^{\sharp}}\right)\right)=\mathcal{R}_{L}^{G}\left(\chi_{\mathcal{E}_{0}^{\sharp}, \phi_{0}^{\sharp}}\right)\right)$. 
1.9. In the rest of this paper, unless otherwise specified, we assume that $q \gg 0$, so that the results of [L90] can be applied. (As mentioned in 0.1 the assumption of loc. cit. on the characteristic of $\mathbf{k}$, can now be removed.) We shall write $R_{M}^{G}: \operatorname{cl}\left(M^{F}\right) \rightarrow \operatorname{cl}\left(G^{F}\right)$ instead of $R_{M, P}^{G}($ with $P \in \Pi(M))$.

Let $(L, S) \in A_{M}, \mathcal{E}_{0}, \phi_{0}$ be as in 1.8. The following result can be deduced from L90, 9.2]:

(a) $\mathcal{R}_{L}^{M}\left(\chi_{\mathcal{E}_{0}^{\sharp}, \phi_{0}^{\sharp}}\right)=R_{L}^{M}\left(\chi_{\mathcal{E}_{0}^{\sharp}, \phi_{0}^{\sharp}}\right)$.

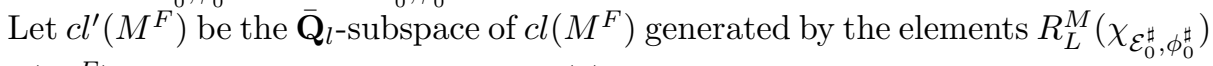
$\in \operatorname{cl}\left(M^{F}\right)$ for various $L, S, \mathcal{E}_{0}, \phi_{0}$ as in (a). The following result will be proved in 乌2.

(b) We have $\operatorname{cl}^{\prime}\left(M^{F}\right)=\operatorname{cl}\left(M^{F}\right)$.

We can now prove 0.1(b). By (b), it is enough to show that if $L, S, \mathcal{E}_{0}, \phi_{0}$ are as in (a) then

(c) $R_{M}^{G}\left(R_{L}^{M}\left(\chi_{\mathcal{E}_{0}^{\sharp}, \phi_{0}^{\sharp}}\right)\right)=\mathcal{R}_{M}^{G}\left(R_{L}^{M}\left(\chi_{\mathcal{E}_{0}^{\sharp}, \phi_{0}^{\sharp}}\right)\right)$.

By (a), the right hand side of (c) is equal to $\mathcal{R}_{M}^{G}\left(\mathcal{R}_{L}^{M}\left(\chi_{\mathcal{E}_{0}^{\sharp}, \phi_{0}^{\sharp}}\right)\right)$ hence, by 1.8 (a), it is equal to $\mathcal{R}_{L}^{G}\left(\chi_{\mathcal{E}_{0}^{\sharp}, \phi_{0}^{\sharp}}\right)$. We have $R_{M}^{G} R_{L}^{M}=R_{L}^{G}: \operatorname{cl}\left(L^{F}\right) \rightarrow \operatorname{cl}\left(G^{F}\right)$. (This is proved in [L76, Cor.5] assuming that $L$ is a maximal torus of $G$; but the same proof works in general.) Thus (c) is equivalent to the equality

$R_{L}^{G}\left(\chi_{\mathcal{E}_{0}^{\sharp}, \phi_{0}^{\sharp}}\right)=\mathcal{R}_{L}^{G}\left(\chi_{\mathcal{E}_{0}^{\sharp}, \phi_{0}^{\sharp}}\right)$

and this follows from (a) (with $M$ replaced by $G$ ). This proves 0.1 (b).

\section{Proof of 1.9(B)}

2.1. Let $\tilde{G}$ be a reductive connected group over $\mathbf{k}$ with an $F_{q}$-rational structure (with Frobenius map $F: \tilde{G} \rightarrow \tilde{G}$ ) such that $\tilde{G}_{d e r}$ is simply connected; assume that we are given a surjective homomorphism of algebraic groups $\tau: \tilde{G} \rightarrow G$ defined over $F_{q}$ whose kernel $K$ is a central torus in $\tilde{G}$. Then $\tilde{M}=\tau^{-1}(M) \in \Lambda(\tilde{G})$ is defined over $F_{q}$. Let $P \in \Pi(M)$ and let $V$ be the unipotent radical of $P$. Then $\tilde{P}=\tau^{-1}(P) \in \Pi(\tilde{M})$. Let $\tilde{V}$ be the unipotent radical of $\tilde{P}$. Let $X=\{g V \in$ $\left.G ; g^{-1} F(g) \in F(V)\right\}, \tilde{X}=\left\{\tilde{g} \tilde{V} \in \tilde{G} ; \tilde{g}^{-1} F(\tilde{g}) \in F(\tilde{V})\right\}$. Now $G^{F} \times M^{F}$ acts on $X$ by $\left(g_{0}, m_{0}\right): g \mapsto g_{0} g m_{0}^{-1}$ and this induces an action of $G^{F} \times M^{F}$ on the $l$-adic cohomology $H_{c}^{i}\left(X, \overline{\mathbf{Q}}_{l}\right)$. Similarly, $\tilde{G}^{F} \times \tilde{M}^{F}$ acts on $H_{c}^{i}\left(\tilde{X}, \overline{\mathbf{Q}}_{l}\right)$. For $u \in G^{F}$ unipotent and for $u^{\prime} \in M^{F}$ unipotent we set $\gamma_{M, V}^{G}\left(u, u^{\prime}\right)=\sum_{i}(-1)^{i} \operatorname{tr}\left(\left(u, u^{\prime}\right), H_{c}^{i}\left(X, \overline{\mathbf{Q}}_{l}\right)\right)$. Similarly for $\tilde{u} \in \tilde{G}^{F}$ unipotent and for $\tilde{u}^{\prime} \in \tilde{M}^{F}$ unipotent we set $\gamma_{\tilde{M}, \tilde{V}}^{\tilde{G}}\left(\tilde{u}, \tilde{u}^{\prime}\right)=$ $\sum_{i}(-1)^{i} \operatorname{tr}\left(\left(\tilde{u}, \tilde{u}^{\prime}\right), H_{c}^{i}\left(\tilde{X}, \overline{\mathbf{Q}}_{l}\right)\right)$. Assuming that $u=\tau(\tilde{u}), u^{\prime}=\tau\left(\tilde{u}^{\prime}\right)$ we show:

(a) $\gamma_{\tilde{M}}^{\tilde{G}}\left(\tilde{u}, \tilde{u}^{\prime}\right)=\left|K^{F}\right| \gamma_{M}^{G}(u, v)$.

The restriction of $\tau: \tilde{G} \rightarrow G$ defines a map $\tilde{X} \rightarrow X$ which is a principal covering with group $K^{F}$. Hence we can identify $H_{c}^{i}\left(X, \overline{\mathbf{Q}}_{l}\right)$ with the $K^{F}$-invariants in $H_{c}^{i}\left(\tilde{X}, \overline{\mathbf{Q}}_{l}\right)$. It follows that (a) can be restated as follows:

$\sum_{i}(-1)^{i} \operatorname{tr}\left(\left(\tilde{u}, \tilde{u}^{\prime}\right), H_{c}^{i}\left(\tilde{X}, \overline{\mathbf{Q}}_{l}\right)\right)=\sum_{k \in K^{F}} \sum_{i}(-1)^{i} \operatorname{tr}\left(\left(k \tilde{u}, \tilde{u}^{\prime}\right), H_{c}^{i}\left(\tilde{X}, \overline{\mathbf{Q}}_{l}\right)\right)$.

By the fixed point formula [DL, 3.2] we have $\sum_{i}(-1)^{i} \operatorname{tr}\left(\left(k \tilde{u}, \tilde{u}^{\prime}\right), H_{c}^{i}\left(\tilde{X}, \overline{\mathbf{Q}}_{l}\right)\right)=0$ for any $k \in K^{F}-\{1\}$ (since the fixed point of translation by $k$ on $\tilde{X}$ is empty). The desired equality follows.

We shall now omit the symbol $V$ in $\gamma_{M, V}^{G}\left(u, u^{\prime}\right)$; we write instead $\gamma_{M}^{G}\left(u, u^{\prime}\right)$. 
2.2. In the setup of 2.1 we define $a: \operatorname{cl}\left(\tilde{G}^{F}\right) \rightarrow \operatorname{cl}\left(G^{F}\right)$ and $a^{\prime}: \operatorname{cl}\left(\tilde{M}^{F}\right) \rightarrow \operatorname{cl}\left(M^{F}\right)$ by

$$
\begin{aligned}
(a \tilde{f})(g) & =\sum_{h \in \tilde{G}^{F} ; \tau(h)=g} \tilde{f}(h), \\
\left(a^{\prime} \tilde{f}\right)(g) & =\sum_{h \in \tilde{M}^{F} ; \tau(h)=g} \tilde{f}(h) .
\end{aligned}
$$

For any $f \in \operatorname{cl}\left(\tilde{M}^{F}\right)$ we show:

$$
a\left(R_{\tilde{M}, \tilde{P}}^{\tilde{G}}(f)\right)=R_{M, P}^{G}\left(a^{\prime}(f)\right) .
$$

We must show that for $g \in G^{F}$ we have

$$
\sum_{h \in \tilde{G}^{F} ; \tau(h)=g} R_{\tilde{M}}^{\tilde{G}}(f)(h)=R_{M}^{G}\left(a^{\prime}(f)\right)(g)
$$

or (using [L90, 1.7(b)]) that

$$
\sum_{\substack{h \in \tilde{G}^{F} ; \\ \tau(h)=g}}\left|\tilde{M}^{F}\right|^{-1}\left|\tilde{G}_{h_{s}}^{0 F}\right|^{-1} \sum_{\substack{z \in \tilde{G}^{F} ; \\ z^{-1} h_{s} z \in \tilde{M}}} \sum_{\substack{\tilde{v} \in z \tilde{M} z^{-1} \cap \tilde{G}_{h_{s}}^{0 F} \\ ; \text { unip. }}} \gamma_{z \tilde{M} z^{-1} \cap \tilde{G}_{h_{s}}^{0}}^{\tilde{G}_{h^{\prime}}^{0}}\left(h_{u}, \tilde{v}\right) f\left(z^{-1} h_{s} \tilde{v} z\right)
$$

(b)

$$
=\left|M^{F}\right|^{-1}\left|G_{g_{s}}^{0 F}\right|^{-1} \sum_{\substack{x \in G^{F} ; \\ x i^{-1} g_{s} x \in M}} \sum_{\substack{v \in x x^{-1} \cap G_{g_{s}}^{0 F} ; \\ \text { unip. }}} \sum_{\substack{\tilde{m} \in \tilde{M}^{F} ; \\ \tau(\tilde{m})=x^{-1} g_{s} v x}} \gamma_{x M^{-1} \cap G_{g_{s}}^{0}}^{G_{g_{s}}^{0}}\left(g_{u}, v\right) f(\tilde{m}) .
$$

The right hand side of (b) is

$$
\begin{aligned}
& \left|K^{F}\right|^{-1} \sum_{\substack{h \in \tilde{G}^{F} ; \\
\tau(h)=g}}\left|\tilde{M}^{F}\right|^{-1}\left|\tilde{G}_{h_{s}}^{0 F}\right|^{-1}\left|K_{F}\right|^{2}\left|K^{F}\right|^{-1} \sum_{\substack{z \in \tilde{G}^{F} ; \\
z^{-1} h_{s} z \in \tilde{M}}} \sum_{\substack{\tilde{v} \in \tilde{M} z^{-1} \cap \tilde{G}_{h_{s}}^{0 F} ; \\
\text { unip. }}} \\
& \sum_{\substack{\tilde{m} \in \tilde{M}^{F} ; \\
\tau(\tilde{m})=\tau\left(z^{-1} h_{s} \tilde{v} z\right)}}\left|K^{F}\right|^{-1} \gamma_{z \tilde{M} z^{-1} \cap \tilde{G}_{h_{s}}^{0}}^{\tilde{G}_{h_{s}}^{0}}\left(h_{u}, \tilde{v}\right) f(\tilde{m}) \\
& =\left|K^{F}\right|^{-1} \sum_{\substack{h \in \tilde{G}^{F} ; \\
\tau(h)=g}}\left|\tilde{M}^{F}\right|^{-1}\left|\tilde{G}_{h_{s}}^{0 F}\right|^{-1}\left|K_{F}\right|^{2}\left|K^{F}\right|^{-1} \\
& \sum_{\substack{z \in \tilde{G}^{F} ; \\
z^{-1} h_{s} z \in \tilde{M}}} \sum_{\substack{\tilde{v} \in z \tilde{M} z^{-1} \cap \tilde{G}_{h_{s}}^{0 F} ; k \in K^{F} \\
\text { unip. }}}\left|K^{F}\right|^{-1} \gamma_{z \tilde{M}_{s} z^{-1} \cap \tilde{G}_{h_{s}}^{0}}^{\tilde{G}_{h_{s}}^{0}}\left(h_{u}, \tilde{v}\right) f\left(k z^{-1} h_{s} \tilde{v} z\right) .
\end{aligned}
$$

(We have used 2.1(a).) This is the same as the left hand side of (b). This proves (a).

2.3. We prove 1.9(b) for $G$ instead of $M$. Let $\Theta_{G}$ be the set of all pairs $(D, \mathcal{X})$ where $D$ is a conjugacy class of $G$ and $\mathcal{X} \in l s_{G}(D)$ is irreducible (up to isomorphism). Now $F$ acts on $\Theta_{G}$ by $F(D, \mathcal{X})=\left(F D, F^{*} \mathcal{X}\right)$. For $(D, \mathcal{X}) \in \Theta_{G}^{F}$ we denote by $\mathcal{L}_{D, \mathcal{X}}$ the line in $\operatorname{cl}\left(G^{F}\right)$ containing the function $\chi_{\mathcal{X}^{\sharp}, \phi^{\sharp}}: G^{F} \rightarrow \overline{\mathbf{Q}}_{l}$ where $\phi: F^{*} \mathcal{X} \stackrel{\sim}{\rightarrow} \mathcal{X}$ is an isomorphism; note that $\chi_{\mathcal{X}^{\sharp}, \phi^{\sharp}}$ is equal to 0 outside the closure of $D$. (This line is well defined.) It is well known and easy to see that

$$
\operatorname{cl}\left(G^{F}\right)=\oplus_{(D, \mathcal{X}) \in \Theta_{G}^{F}} \mathcal{L}_{D, \mathcal{X}} .
$$


Hence to prove that $\operatorname{cl}^{\prime}\left(G^{F}\right)=\operatorname{cl}\left(G^{F}\right)$ it is enough to show that

(a) if $(D, \mathcal{X}) \in \Theta_{G}^{F}$ and $\phi: F^{*} \mathcal{X} \stackrel{\sim}{\rightarrow} \mathcal{X}, f_{0}=\chi_{\mathcal{X}^{\sharp}, \phi^{\sharp}}$, then $f_{0} \in c l^{\prime}\left(G^{F}\right)$.

In the special case where $G_{d e r}$ is simply connected, this follows from [L90, 9.5]. We shall deduce the general case from this special case. We can find $\tau: \tilde{G} \rightarrow G, F$ : $\tilde{G} \rightarrow \tilde{G}, K$ as in 2.1 such that $\tilde{G}_{d e r}$ is simply connected. Let $a: \operatorname{cl}\left(\tilde{G}^{F}\right) \rightarrow \operatorname{cl}\left(G^{F}\right)$ be as in 2.2. We define a linear map $b: \operatorname{cl}\left(G^{F}\right) \rightarrow \operatorname{cl}\left(\tilde{G}^{F}\right)$ by $(b f)(\tilde{g})=f(\tau(\tilde{g}))$; for $f \in \operatorname{cl}\left(G^{F}\right)$ we have $a b f=\left|K^{F}\right| f$. Since 1.9(b) holds for $\tilde{G}$, we have $b f_{0} \in c l^{\prime}\left(\tilde{G}^{F}\right)$ hence $\left|K^{F}\right| f_{0}=a b f_{0} \in a\left(c l^{\prime}\left(\tilde{G}^{F}\right)\right)$. Thus it is enough to show that $a\left(c l^{\prime}\left(\tilde{G}^{F}\right)\right) \subset$ $c l^{\prime}\left(G^{F}\right)$.

Let $(\tilde{L}, \tilde{S}) \in A_{\tilde{G}}$ be such that $F(\tilde{L})=\tilde{L}, F(\tilde{S})=\tilde{S}$ and let $\mathcal{F} \in l s_{\tilde{L}}(\tilde{S})$ be irreducible cuspidal with a given isomorphism $\psi: F^{*} \mathcal{F} \stackrel{\sim}{\rightarrow} \mathcal{F}$. It is enough to show that

(b) $a\left(R_{\tilde{L}}^{\tilde{G}}\left(\chi_{\mathcal{F}^{\sharp}, \psi^{\sharp}}\right)\right) \in c l^{\prime}\left(G^{F}\right)$.

Let $L=\tau(\tilde{L}), S=\tau(\tilde{S})$; we have $(L, S) \in A_{M}$. Let $\tau^{\prime}: \tilde{L} \rightarrow L$ be the restriction of $\tau$; we define define $a^{\prime}: \operatorname{cl}\left(\tilde{L}^{F}\right) \rightarrow \operatorname{cl}\left(L^{F}\right)$ by $\left(a^{\prime} \tilde{f}\right)(g)=\sum_{\tilde{g} \in \tilde{L}^{F} ; \tau^{\prime}(\tilde{g})=g} \tilde{f}(\tilde{g})$. By 2.2(a), for any $f \in \operatorname{cl}\left(\tilde{L}^{F}\right)$ we have

(c) $a\left(R_{\tilde{L}}^{\tilde{G}}(f)\right)=R_{L}^{G}\left(a^{\prime}(f)\right)$.

From this we see that the left hand side of (b) is equal to $R_{L}^{G}\left(a^{\prime}\left(\chi_{\mathcal{F}^{\sharp}, \psi^{\sharp}}\right)\right)$. From the definitions we see that $a^{\prime}\left(\chi_{\mathcal{F}^{\sharp}, \psi^{\sharp}}\right)$ is a linear combination of functions of the form $\chi_{\mathcal{E}_{0}^{\sharp}, \phi_{0}^{\sharp}}: L^{F} \rightarrow \overline{\mathbf{Q}}_{l}$ where $\mathcal{E}_{0} \in l s_{L}(S)$ is irreducible cuspidal and $\phi_{0}: F^{*} \mathcal{E}_{0} \stackrel{\sim}{\rightarrow} \mathcal{E}_{0}$. It follows that $R_{L}^{G}\left(a^{\prime}\left(\chi_{\mathcal{F}^{\sharp}, \psi^{\sharp}}\right)\right) \in c l^{\prime}\left(G^{F}\right)$. We see that (b) holds. This completes the proof of 1.9(b) for $G$.

\section{A DiRECT SUm DECOMposition of $c l\left(G^{F}\right)$}

3.1. In this section there is no restriction on $q$. Let $(D, \mathcal{X}) \in \Theta_{G}$. We associate to $(D, \mathcal{X})$ an admissible stratum of $G$. Let $E$ be the set of semisimple parts of elements in $D$; this is a conjugacy class in $G$. For $s \in E$ let $[s]$ be the set of unipotent conjugacy classes of $G_{s}^{0}$ such that $s C \subset D$. For any $s \in E$ and $C \in[s]$ we define $f_{s}: C \rightarrow D$ by $u \mapsto s u$; then $f_{s}^{*} \mathcal{X} \in l s_{G_{s}^{0}}(C)$. Let $f_{s}^{*} \mathcal{X}=\oplus \mathcal{Y} \in Q_{s, C} \mathcal{Y}$ be the isotypic decomposition of $f_{s}^{*} \mathcal{X}$; thus each $\mathcal{Y}$ is an isotypic object of $l s_{G_{s}^{0}}(C)$. Let $D^{\prime}$ be the set of all pairs $(g, \mathcal{Y})$ where $g \in D$ and $\mathcal{Y} \in Q_{g_{s}, C}$ where $C \in\left[g_{s}\right]$ contains $g_{u}$. Then $D^{\prime}$ is naturally an algebraic variety with a transitive action of $G$ such that the map $D^{\prime} \rightarrow D,(g, \mathcal{Y}) \mapsto g$ is a $G$-equivariant unramified finite covering. For $s \in E, C \in[s], \mathcal{Y} \in Q_{s, C}$, we choose an irreducible summand $\eta$ of $\mathcal{Y}$; the generalized Springer correspondence [L84, 6.3] for the reductive connected group $G_{s}^{0}$ associates to the pair $(C, \eta)$ a triple $(L, S, \mathcal{F})=\left(L \mathcal{Y}, S_{\mathcal{Y}}, \mathcal{F}_{\mathcal{Y}}\right)$ (up to $G_{s}^{0}$-conjugacy) where $L \in \Lambda\left(G_{s}^{0}\right), S=\mathcal{Z}_{L}^{0} c$ with $c=c \mathcal{Y}$ a unipotent class of $L$ and $\mathcal{F}=\overline{\mathbf{Q}}_{l} \otimes \mathcal{F}_{0} \in l s_{L}(S)$ is irreducible cuspidal with $\mathcal{F}_{0} \in l s_{L}(c)$ irreducible; this triple is independent of the choice of $\eta$ since $\mathcal{Y}$ is isotypic. Let $M=M_{\mathcal{Y}}=Z_{G}\left(\mathcal{Z}_{L}^{0}\right) \in \Lambda(G)$. Let $D_{\mathcal{Y}}$ be the conjugacy class in $M$ containing $s c$. Let $\Sigma=\Sigma_{\mathcal{Y}}=D_{\mathcal{Y}} \mathcal{Z}_{M}^{0}$. Since $L \in \Lambda\left(G_{s}^{0}\right)$, we have $Z_{G_{s}^{0}}\left(\mathcal{Z}_{L}^{0}\right)=L$ hence $\left(Z_{G_{s}}\left(\mathcal{Z}_{L}^{0}\right)\right)^{0}=L$. We have $M_{s}=G_{s} \cap M=G_{s} \cap Z_{G}\left(\mathcal{Z}_{L}^{0}\right)=$ $Z_{G_{s}}\left(\mathcal{Z}_{L}^{0}\right)$ so that $M_{s}^{0}=L$. We have $Z_{M}\left(\mathcal{Z}_{M_{s}^{0}}^{0}\right)=Z_{M}\left(\mathcal{Z}_{L}^{0}\right)=Z_{G}\left(\mathcal{Z}_{L}^{0}\right) \cap M=M$ hence $s$ is isolated in $M$ and $\Sigma$ is an isolated stratum of $M$. Hence we can define $Y=Y_{M, \Sigma}^{G}$, a stratum of $G$. If $(L, S, \mathcal{F})$ is replaced by a $G_{s}^{0}$-conjugate or if $(s, C, \mathcal{Y})$ is replaced by a triple in the same $G$-orbit, then $Y$ is replaced by a $G$-conjugate hence it remains the same. Thus the stratum $Y$ depends only on $(D, \mathcal{X})$. For 
$\mathcal{Y},(L, S, \mathcal{F}), M, \Sigma$ as above we can find $\mathcal{F}^{\prime} \in l s_{M}(\Sigma)$ irreducible such that the inverse image of $\mathcal{F}^{\prime}$ under $C \rightarrow \Sigma, u \mapsto s u$ contains $\mathcal{F}$ as a direct summand. By the arguments in [L84, 2.10], $\mathcal{F}^{\prime}$ is cuspidal. It follows that $Y$ is an admissible stratum. We set $Y=\psi(D, \mathcal{X})$.

Note that if $(D, \mathcal{X}) \in \Theta_{G}^{F}$ then $F(Y)=Y$.

3.2. Let $\Gamma_{G}^{\prime}$ be the set of all triples $\left(L, S, \mathcal{E}_{0}\right)$ where $(L, S) \in A_{G}$ is such that $F L=L, F S=S$ and $\mathcal{E}_{0} \in l s_{L}(S)$ is irreducible cuspidal (up to isomorphism) such that $F^{*} \mathcal{E}_{0} \cong \mathcal{E}_{0}$. Let $\Gamma_{G}$ be the set of orbits of the conjugation action of $G^{F}$ in $\Gamma_{G}^{\prime}$. For $\left(L, S, \mathcal{E}_{0}\right) \in \Gamma_{G}^{\prime}$ we choose an isomorphism $\phi_{0}: F^{*} \mathcal{E}_{0} \stackrel{\sim}{\longrightarrow} \mathcal{E}_{0}$ of local systems on $S$. Then $\chi_{\mathcal{E}_{0}^{\sharp}, \phi_{0}^{\sharp}}: L^{F} \rightarrow \overline{\mathbf{Q}}_{l}$ is well defined; it is a class function on $L^{F}$. Let $\mathcal{L}_{L, S, \mathcal{E}_{0}}$ be the line in $\operatorname{cl}\left(G^{F}\right)$ containing $\mathcal{R}_{L}^{G}\left(\chi_{\mathcal{E}_{0}^{\sharp}, \phi_{0}^{\sharp}}\right)$ for some/any $\phi_{0}$ as above; this line depends only on the image of $\left(L, S, \mathcal{E}_{0}\right)$ in $\Gamma_{G}$. We have the following result.

\section{Theorem 3.3.}

(i) We have $\operatorname{cl}\left(G^{F}\right)=\oplus_{\left(L, S, \mathcal{E}_{0}\right) \in \Gamma_{G}} \mathcal{L}_{L, S, \mathcal{E}_{0}}$.

(ii) For any $F$-stable admissible stratum $Y$ of $G$ we define $c_{Y}\left(G^{F}\right)$ to be the subspace $\sum_{\left(L, S, \mathcal{E}_{0}\right) \in \Gamma_{G} ; Y_{L, S}^{G}=Y} \mathcal{L}_{L, S, \mathcal{E}_{0}}$ of $\operatorname{cl}\left(G^{F}\right)$ (this is a direct sum, see (i)); we define $\underline{c l}_{Y}\left(G^{F}\right)$ to be the subspace $\oplus_{(D, \mathcal{X}) \in \Theta_{G}^{F} ; \psi(D, \mathcal{X})=Y} \mathcal{L}_{D, \mathcal{X}}$ of $\operatorname{cl}\left(G^{F}\right)$ (see [2.3, 3.1). We have $c l_{Y}\left(G^{F}\right)=\underline{c l}_{Y}\left(G^{F}\right)$ and $c l\left(G^{F}\right)=\oplus_{Y} c l_{Y}\left(G^{F}\right)$ where $Y$ runs over the $F$-stable admissible strata of $G$.

The fact that the sum in (i) is direct follows from the orthogonality relations [L85, 9.9] (its hypotheses are satisfied by the results in [L86] and [L12]). If $(D, \mathcal{X}) \in$ $\Theta_{G}^{F}$ and $Y=\psi(D, \mathcal{X})$ then we have

(a) $\mathcal{L}_{D, \mathcal{X}} \subset \operatorname{cl}_{Y}\left(G^{F}\right)$.

When $G_{d e r}$ is simply connected, (a) follows from [L90, 9.5]. (One can replace $R_{L}^{G}$ in loc.cit. with $q$ large by $\mathcal{R}_{L}^{G}$ without restriction on $q$.) The general case can be reduced to this special case by passage to $\tilde{G}$ as in the proof in 2.3 (again replacing $R_{L}^{G}$ by $\left.\mathcal{R}_{L}^{G}\right)$. Since the lines $\mathcal{L}_{D, \mathcal{X}}$ span $c l\left(G^{F}\right)$ we see that (a) implies that the sum in (i) is equal to $c l\left(G^{F}\right)$. Thus (i) holds. From (a) we see that $\underline{c l}_{Y}\left(G^{F}\right) \subset \operatorname{cl}_{Y}\left(G^{F}\right)$ for any $Y$. Since $\oplus_{Y} \underline{c l}_{Y}\left(G^{F}\right)=\oplus_{Y} c l_{Y}\left(G^{F}\right)=\operatorname{cl}\left(G^{F}\right)$ (see 2.3 and (i)) it follows that $\underline{c l}_{Y}\left(G^{F}\right)=c l_{Y}\left(G^{F}\right)$ for any $Y$. This proves (ii).

3.4. From 3.3 and the orthogonality relations mentioned in the proof of 3.3 one can deduce that the "Mackey formula" for $R_{L, P}^{G}$ stated by Deligne (unpublished) in 1976 for $q$ large and in $[\mathrm{BM}$ for $q>2$ remains valid without restriction on $q$ if $R_{L, P}^{G}$ is replaced by $\mathcal{R}_{L}^{G}$.

3.5. Let $(D, \mathcal{X}) \in \Theta_{G}$. We use notation of 3.1. We say that $(D, \mathcal{X})$ is of principal type if for $s \in E, C \in[s]$, the local system $f_{s}^{*} \mathcal{X}$ on $C$ is such that some/any irreducible summand $\eta$ of $f_{s}^{*} \mathcal{X}$ is such that $(C, \eta)$ appears in the usual Springer correspondence for $G_{s}^{0}$. An equivalent condition is that the stratum $Y=\psi(D, \mathcal{X})$ is the variety of regular semisimple elements in $G$. For example, $\left(D, \overline{\mathbf{Q}}_{l}\right)$ is of principal type.

Now let $(D, \mathcal{X}) \in \Theta_{G}^{F}$ be of principal type; let $\phi: F^{*} \mathcal{X} \stackrel{\sim}{\rightarrow} \mathcal{X}$ be an isomorphism. From 3.3(a) we deduce

$$
\chi_{\mathcal{X}^{\sharp}, \phi^{\sharp}}=\sum_{T, \theta} c_{D, \mathcal{X} ; T, \theta} \mathcal{R}_{T}^{G}(\theta)
$$


where $T$ runs over the $F$-stable maximal tori in $G, \theta$ runs through the set of characters $T^{F} \rightarrow \overline{\mathbf{Q}}_{l}$ and the pairs $(T, \theta)$ are taken up to $G^{F}$-conjugacy; $c_{D, \mathcal{X} ; T, \theta} \in$ $\overline{\mathbf{Q}}_{l}$ are uniquely determined. Equivalently, we have

$$
\chi_{\mathcal{X} \sharp, \phi^{\sharp}}=\sum_{T, \theta} c_{D, \mathcal{X} ; T, \theta} R_{T}^{G}(\theta) .
$$

Indeed, we have $R_{T}^{G}(\theta)=\mathcal{R}_{T}^{G}(\theta)$. This follows from the results in [L90] (for large $q)$ and their extension to general $q$ in $[\mathrm{Sh}$. Moreover, from [L90, 9.5] we see that $c_{D, \mathcal{X} ; T, \theta}$ are explicitly known (at least if $G_{d e r}$ is simply connected, but the general case can be reduced to this case as before). Since the multiplicities of irreducible representations of $G^{F}$ in $R_{T}^{G}(\theta)$ are known, it follows that the functions $\chi_{\mathcal{X}^{\sharp}, \phi^{\sharp}}$ are computable as explicit linear combinations of irreducible characters.

In particular, (a),(b) hold when $D$ is an $F$-stable conjugacy class in $G^{F}$ and $\mathcal{X}=\overline{\mathbf{Q}}_{l}$.

3.6. Let $(D, \mathcal{X}) \in \Theta_{G}^{F}$. Let $\mathcal{Z}$ be the set of all $\left(D^{\prime}, \mathcal{X}^{\prime}\right) \in \Theta_{G}^{F}$ such that $D^{\prime}$ is contained in the closure of $D$. For any $\left(D^{\prime}, \mathcal{X}^{\prime}\right) \in \mathcal{Z}$ we choose an isomorphism $\phi_{\mathcal{X}^{\prime}}: F^{*} \mathcal{X}^{\prime} \stackrel{\sim}{\longrightarrow} \mathcal{X}^{\prime}$. We have

(a) $\chi_{\mathcal{X}, \phi_{\mathcal{X}}}=\sum_{\left(D^{\prime}, \mathcal{X}^{\prime}\right) \in \mathcal{Z}} d_{D^{\prime}, \mathcal{X}^{\prime}} \chi_{\mathcal{X}^{\prime} \sharp, \phi_{\mathcal{X}^{\prime}}^{\sharp}}$

where $d_{D^{\prime}, \mathcal{X}^{\prime}} \in \overline{\mathbf{Q}}_{l}$. Assume now that $(D, \mathcal{X})$ is of principal type. Then $d_{D^{\prime}, \mathcal{X}^{\prime}}=$ 0 unless $\left(D^{\prime}, \mathcal{X}^{\prime}\right)$ is of principal type. (This can be deduced from the results in L86 on Green functions.) Using [3.5(b) we deduce

$$
\chi \mathcal{X}, \phi=\sum_{T, \theta} \tilde{c}_{D, \mathcal{X} ; T, \theta} R_{T}^{G}(\theta)
$$

where $\tilde{c}_{D, \mathcal{X} ; T, \theta} \in \overline{\mathbf{Q}}_{l}$ is explicitly computable. In particular, (b) holds when $\mathcal{X}=\overline{\mathbf{Q}}_{l}$. We see that:

(c) the class function on $G^{F}$ equal to 1 on $D^{F}$ and equal to 0 on $G^{F}-D^{F}$ is a linear combination of functions of the form $R_{T}^{\theta}$.

This has been conjectured in [L78, 2.16]. Note that the coefficients in the linear combination above are explicitly computable. Since each $R_{T}^{\theta}$ is an explicit linear combination of irreducible characters, we deduce that for any $D$ as above the average value on $D^{F}$ of any irreducible character of $G^{F}$ is explicitly computable. In the case where $D$ is a semisimple class, a result like (c) appears (in a stronger form) in [DL, 7.5].

Note that (c) also appears in [DM20, Cor.13.3.5] and in GM20, Cor.2.7.13] (of which the author learned after submitting this paper).

\section{REFERENCES}

[BM] Cédric Bonnafé and Jean Michel, Computational proof of the Mackey formula for $q>2$, J. Algebra 327 (2011), 506-526, DOI 10.1016/j.jalgebra.2010.10.030. MR2746047

[DL] P. Deligne and G. Lusztig, Representations of reductive groups over finite fields, Ann. of Math. (2) 103 (1976), no. 1, 103-161, DOI 10.2307/1971021. MR.393266

[DM20] François Digne and Jean Michel, Representations of finite groups of Lie type, 2nd ed., London Mathematical Society Student Texts, vol. 95, Cambridge University Press, Cambridge, 2020. MR 4211777

[GM20] Meinolf Geck and Gunter Malle, The character theory of finite groups of Lie type: A guided tour, Cambridge Studies in Advanced Mathematics, vol. 187, Cambridge University Press, Cambridge, 2020. MR4211779

[L76] G. Lusztig, On the finiteness of the number of unipotent classes, Invent. Math. 34 (1976), no. 3, 201-213, DOI 10.1007/BF01403067. MR419635 
[L78] George Lusztig, Representations of finite Chevalley groups, CBMS Regional Conference Series in Mathematics, vol. 39, American Mathematical Society, Providence, R.I., 1978. Expository lectures from the CBMS Regional Conference held at Madison, Wis., August 8-12, 1977. MR518617

[L84] G. Lusztig, Intersection cohomology complexes on a reductive group, Invent. Math. 75 (1984), no. 2, 205-272, DOI 10.1007/BF01388564. MR732546

[L85] George Lusztig, Character sheaves. II, III, Adv. in Math. 57 (1985), no. 3, 226-265, 266-315, DOI 10.1016/0001-8708(85)90064-7. MR806210

[L86] George Lusztig, Character sheaves. V, Adv. in Math. 61 (1986), no. 2, 103-155, DOI 10.1016/0001-8708(86)90071-X. MR849848

[L90] George Lusztig, Green functions and character sheaves, Ann. of Math. (2) 131 (1990), no. 2, 355-408, DOI 10.2307/1971496. MR.1043271

[L04] G. Lusztig, Character sheaves on disconnected groups. V, Represent. Theory 8 (2004), 346-376, DOI 10.1090/S1088-4165-04-00251-1. MR2077486

[L12] G. Lusztig, On the cleanness of cuspidal character sheaves (English, with English and Russian summaries), Mosc. Math. J. 12 (2012), no. 3, 621-631, 669, DOI 10.17323/16094514-2012-12-3-621-631. MR3024826

[Sh] Toshiaki Shoji, Character sheaves and almost characters of reductive groups. I, II, Adv. Math. 111 (1995), no. 2, 244-313, 314-354, DOI 10.1006/aima.1995.1024. MR.1318530

Department of Mathematics, M.I.T., Cambridge, Massachusetts 02139

Email address: gyuri@mit.edu 